\title{
Densidade de estocagem e parâmetros eritrocitários de pacus criados em tanques-rede
}

\author{
Fábio Bittencourt ${ }^{1}$, Aldi Feiden ${ }^{2}$, Arcangelo Augusto Signor ${ }^{1}$, Wilson Rogério Boscolo ${ }^{2}$, \\ Evandro Kleber Lorenz ${ }^{1}$, Márcia Luzia Ferrarezi Maluf ${ }^{2}$
}

\footnotetext{
1 Universidade Estadual do Oeste do Paraná - UNIOESTE/Campus de Marechal Cândido Rondon.

2 Universidade Estadual do Oeste do Paraná - UNIOESTE/Campus de Toledo.
}

RESUMO - O objetivo neste trabalho foi avaliar o manejo, as características eritrocitárias e o nível de glicose sanguínea de pacus (Piaractus mesopotamicus) estocados em diferentes densidades em tanques-rede. Foram utilizados 2.700 peixes com peso inicial de $142,11 \pm 10,54 \mathrm{~g}$, distribuídos em nove tanques-rede de $5 \mathrm{~m}^{3}$ de volume útil durante 240 dias. O delineamento utilizado foi o inteiramente casualizado, com três densidades (200, 300 e 400 peixes por tanque), cada uma com três repetições. Somente o peso final e o ganho de peso diminuíram com o aumento da densidade de estocagem. A biomassa final aumenta de acordo com o número de animais estocados por unidade produtiva. As densidades de criação não influenciaram o rendimento de carcaça, mas afetaram o comprimento total, que foi maior nos peixes criados em baixa densidade, que não diferiram daqueles cultivados em densidade intermediária. Os peixes criados em menor densidade apresentaram maior deposição lipídica, mas não diferiram significativamente daqueles estocados em densidade intermediária. As variáveis eritrocitárias, como eritrócitos totais, hemoglobina, hematócrito, volume corpuscular médio e concentrações de hemoglobina corpuscular média e de hemoglobina corpuscular média, não apresentaram diferenças estatísticas entre as densidades utilizadas e permaneceram na faixa normal para a espécie. Os níveis de glicose no sangue não diferiram significativamente entre as densidades trabalhadas. A densidade de estocagem não influencia os parâmetros eritrocitários, o nível de glicose nem o rendimento de carcaça de pacus. A densidade de 200 peixes/tanque resulta em maior peso final e ganho de peso, porém reduz a produtividade por área e aumenta a deposição lipídica dos peixes.

Palavras-chave: criação intensiva, desempenho, espécie nativa, parâmetros hematológicos, Piaractus mesopotamicus

\section{Stocking density and erythrocytic parameters of pacu raised in cages}

ABSTRACT - The aim of this study was to evaluate management, erythrocyte features and blood glucose level of pacu (Piaractus mesopotamicus), stocked at different densities in cages. It was used 2,700 fish with initial average weight of $142.11 \pm 10.54 \mathrm{~g}$ distributed in nine cages of $5 \mathrm{~m}^{3}$ of useful volume during 240 days. A complete random design was used, with three densities (200, 300 and 400 fish per cage), each one with three replicates. Only final weight and weight gain decreased with the increase of stocking density. Final biomass increase according to the number of animals stocked per productive unit Densities of farming did not influence carcass yield, but they affected total length, which was higher in fish raised in low density, which did not differ from those raised in intermediate density. Fish raised in lower density showed the greatest lipid deposition, but they did not significantly differ from those stocked in intermediate density. Erythrocyte variables like total erythrocytes, hemoglobin, hematocrit, average corpuscular volume, average corpuscular hemoglobin concentrations and average corpuscular hemoglobin did not present statistical differences among the used densities and they remain in the normal range for the species. Levels of glucose in the blood did not differ significantly among the analyzed densities. Stocking densities do not influence erythrocyte parameters, glucose level neither carcass yields of pacu fish. Density 200 fish/cage results in higher final weight and weight gain; however, it reduces productivity per area and increases lipid deposition of the fish.

Key Words: hematological parameters, intensive rearing, native species, performance, Piaractus mesopotamicus

\section{Introdução}

O Brasil possui inúmeras espécies de peixes autóctones com potencial zootécnico para a criação em tanques-rede. Entre elas, destaca-se o pacu, Piaractus mesopotamicus (Holmberg, 1887 inserir nas referências), que é representante da superordem Ostariophysi, cujos peixes são os de maior valor comercial na pesca e na piscicultura brasileira (Urbinati \& Gonçalves, 2005).

Muitos fatores podem interferir no período de criação desses peixes. Entre esses fatores, destaca-se a densidade de estocagem, que pode afetar diretamente o crescimento. 
Segundo Brandão et al. (2004), para o desenvolvimento de um manual de criação para uma espécie de peixe, o primeiro passo é a definição da densidade de estocagem ideal, na qual possam ser atingidos os níveis ótimos de produtividade.

Normalmente, peixes criados em baixas densidades de estocagem apresentam boa taxa de crescimento e alta porcentagem de sobrevivência, porém com baixa produção por área (Gomes et al., 2000), caracterizando baixo aproveitamento da área disponível. Por sua vez, peixes mantidos em altas densidades normalmente têm menor crescimento (El-Sayed, 2002), ficam estressados (Iguchi et al., 2003) e sujeitos ao aparecimento de interações sociais que levam à produção com tamanho heterogêneo em mesmo lote (Cavero et al., 2003), o que não é interessante na produção comercial.

A intensificação dos sistemas de produção tem como propósito alcançar índices de produtividade elevados em menor espaço físico e em menor tempo de criação (Ono \& Kubitza, 1999). Essas características favorecem a ocorrência de problemas sanitários, pois o transporte, a má qualidade da água, a alta densidade de estocagem, entre outros, são fatores estressantes, que reduzem os mecanismos de defesa orgânica e aumentam a susceptibilidade dos peixes a enfermidades, prejudicando seriamente a produção (Schalch et al., 2005). Segundo Ranzani-Paiva et al. (1999), os exames hematológicos são necessários para se caracterizar fisiologicamente uma espécie em seu ambiente natural e auxiliar no manejo das criações, relacionando os resultados à presença de infestações ou infecções nos animais, ou mesmo às alterações ambientais.

Assim, realizou-se este trabalho com o objetivo de avaliar a influência de três densidades de criação no desempenho zootécnico, na composição centesimal, nos níveis de glicose sanguínea e nos parâmetros eritrocitários de pacus (P. mesopotamicus) criados em tanques-rede.

\section{Material e Métodos}

O experimento foi realizado no período de julho de 2007 a março de 2008 (240 dias de experimento), na área de transição do reservatório da Itaipu Binacional, no Centro de Desenvolvimento de Tecnologias para Piscicultura em Tanque-rede, localizado no Refúgio Biológico do município de Santa Helena, Paraná, entre as coordenadas geográficas

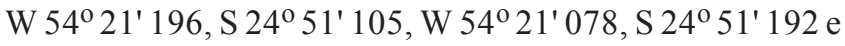

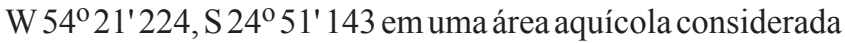
oligotrófica (Bueno et al., 2008). Utilizaram-se 2.700 peixes com peso inicial de 142,11 $\pm 10,54 \mathrm{~g}$ estocados em nove tanques-rede confeccionados com arame galvanizado revestido de polietilieno, com dimensões de 2,0 $\times 2,0 \times 1,5 \mathrm{~m}$ (comprimento largura $\times$ altura), totalizando $5 \mathrm{~m}^{3}$ de volume útil. Os tanques-rede foram dispostos em uma linha da área aquícola, separados por $2 \mathrm{~m}$ de distância em um ambiente com profundidade média de $8 \mathrm{~m}$.

O delineamento utilizado foi o inteiramente casualizado, com três densidades de criação $(200,300$ e 400 peixes por tanque, ou seja, 40,60 e 80 peixes. $\mathrm{m}^{-3}$ ), cada uma com três repetições. Os animais receberam ração comercial extrusada contendo $32 \%$ de proteína bruta até a saciedade aparente dos animais, três vezes ao dia ( $9 \mathrm{~h}, 13 \mathrm{~h}$ e às $17 \mathrm{~h})$.

Para os manejos mensais de biometria, utilizou-se uma balsa com sistema de talha para içar os tanques-rede. Com auxílio de um puçá, uma amostra de aproximadamente $10 \%$ dos animais de cada unidade produtiva foi retirada para pesagem.

Os parâmetros físicos e químicos da água, como temperatura e transparência, foram mensurados duas vezes ao dia, pela manhã e à tarde, com auxílio de um termômetro e disco de Secchi, respectivamente, enquanto o $\mathrm{pH}$, o oxigênio dissolvido e a condutividade elétrica foram medidos semanalmente utilizando-se potenciômetros digitais.

Ao final do período experimental, os animais foram mantidos em jejum por 24 horas, e dez peixes de cada unidade experimental foram coletados para coleta de sangue. Os animais foram anestesiados com Eugenol ${ }^{\circledR}$ (solução de óleo de cravo) na dose de $60 \mathrm{mg} . \mathrm{L}^{-1}$. Em seguida, por punção caudal, foram coletados, com o auxílio de uma seringa descartável contendo EDTA (10\%), $2 \mathrm{~mL}$ de sangue de cada animal. Essa alíquota foi destinada à contagem do número de eritrócitos em câmara de Neubauer com microscópio óptico com objetiva de 40 vezes após a diluição do sangue com líquido de Hayem (Collier, 1944). As determinações de hemoglobina e hematócrito foram realizadas segundo metodologia descrita por Collier (1944) e Goldenfarb et al. (1971), respectivamente. Para avaliação bioquímica da glicose, utilizou-se o plasma colhido com fluoreto e separado por centrifugação e procedeu-se à leitura em espectrofotômetro.

Nas análises hematológicas, avaliaram-se os níveis de glicose e hemoglobina, o hematócrito, o eritrócito, o volume corpuscular médio e as concentrações de hemoglobina corpuscular média.

Após a coleta de sangue, os animais foram retirados dos tanques-rede para a avaliação do peso final, do ganho de peso, da biomassa final, da taxa de sobrevivência e da conversão alimentar.

Posteriormente, dez animais de cada tanque-rede foram retirados aleatoriamente e insensibilizados por choque térmico, acondicionados em caixa com gelo e transportados ao Laboratório de Tecnologia do Pescado da Universidade Estadual do Oeste do Paraná - UNIOESTE, Campus de 
Toledo, para avaliação dos seguintes índices de rendimento da carcaça: comprimento total, comprimento padrão, peso total, peso eviscerado, tronco limpo, filé, cabeça, gordura visceral e índice hepatossomático (Tabela 2). Para tanto, os animais foram pesados em balança digital, abertos na região ventral e eviscerados. Em seguida, foram efetuados cortes referentes para separação da cabeça e das nadadeiras e retirada de couro e filé. As vísceras foram colocadas em placas de Petry - onde foi feita separação da gordura e do fígado - e o material resultante foi pesado em balança analítica.

As amostras dos filés foram separadas para análise da composição química, segundo metodologia descrita na AOAC (2005), e avaliação da umidade e dos teores de proteína bruta, extrato etéreo e matéria mineral.

Os dados foram tabulados e submetidos à análise de variância e, quando constatadas diferenças significativas, foram submetidos ao teste Tukey a $5 \%$ de probabilidade pelo programa estatístico SAEG.

\section{Resultados e Discussão}

Os parâmetros de qualidade da água durante o período experimental foram de 55,52 $\pm 6,24 \mu \mathrm{S} . \mathrm{cm}^{-1}$; $7,27 \pm 0,09 \mathrm{mg} . \mathrm{L}^{-1}$ e 7,45 $\pm 0,05$, respectivamente, para condutividade elétrica, oxigênio dissolvido e potencial hidrogeniônico. A temperatura média da água entre os meses de julho de 2007 e março de 2008 foi de $24,32 \pm 3,79$ e $25,15 \pm 4,28^{\circ} \mathrm{C}$ nos turnos matutino e vespertino, respectivamente. A transparência da água durante todo o período de produção foi de $2,51 \pm 0,37$ e 2,96 $\pm 0,43 \mathrm{~m}$ pela manhã e à tarde, respectivamente, valores que estão de acordo com aqueles recomendados para a criação de peixes de clima tropical (Boyd, 1990; Sipaúba-Tavares, 1995).

$\mathrm{Na}$ avaliação do desempenho zootécnico dos peixes ao final de 240 dias de criação, verificou-se que o aumento da densidade de estocagem proporcionou redução nos valores de peso final e ganho de peso (Tabela 1). Esse resultado

Tabela 1 - Desempenho produtivo de pacus em tanques-rede em três diferentes densidades de criação

\begin{tabular}{lcccc}
\hline Variável $^{1}$ & \multicolumn{3}{c}{ Densidade } & C V \\
\cline { 2 - 4 } & 200 & 300 & 400 & \\
\hline Peso inicial (g) & 148,66 & 145,33 & 132,33 & 6,04 \\
Peso final (g) & $1258,06 \mathrm{a}$ & $1051,00 \mathrm{~b}$ & $943,80 \mathrm{c}$ & 2,45 \\
Ganho de peso (g) & $1109,40 \mathrm{a}$ & $905,66 \mathrm{~b}$ & $811,40 \mathrm{c}$ & 3,56 \\
Biomassa final (kg) & $247,00 \mathrm{c}$ & $316,09 \mathrm{~b}$ & $376,68 \mathrm{a}$ & 3,34 \\
Sobrevivência (\%) & 98,00 & 99,66 & 98,91 & 1,54 \\
Conversão alimentar & 2,58 & 2,43 & 2,67 & 5,27 \\
\hline${ }^{1}$ Médias na mesma linha seguidas de letras distintas diferem entre si pelo teste \\
Tukey a 5\%. \\
CV= coeficiente de variação.
\end{tabular}

corrobora aquele observado por Marengoni (2006), que, trabalhando com tilápia-do-nilo (Oreochromis niloticus) nas densidades de 250,300,350 e 400 peixes. $\mathrm{m}^{-3}$, observou maiores valores de peso final e ganho de peso em tanquesrede com menor número de indivíduos por unidade de área. Nesse mesmo sentido, Silva et al. (2002) estudaram o efeito da densidade de estocagem $\left(90,120\right.$ e 150 peixes.tanque $\left.{ }^{-1}\right)$ e das trocas de água (30 e 60 min) na criação de tilápias-denilo (O. niloticus) em "raceway" e encontraram maiores valores de peso final e ganho de peso nos peixes criados em menor densidade.

Os resultados de peso final e ganho de peso parecem estar diretamente ligados à fase de desenvolvimento dos animais e à espécie. Coulibaly et al. (2006) estudaram o efeito da densidade de estocagem em duas fases de desenvolvimento na criação do catfish africano (Heterobranchus longifilis) em tanques-rede. Primeiramente, constataram que os animais em estágio inicial de desenvolvimento, cultivados em densidade de 50, 100, 200,500 e 1000 peixes. $\mathrm{m}^{-3}$ por 90 dias, não apresentaram diferenças estatísticas no peso final e ganho de peso diário entre as densidades estudadas. Por outro lado, na fase de crescimento, as menores densidades $(6,12,25,50$ e 100 peixes. $\mathrm{m}^{-3}$ ) resultaram nos maiores valores de peso final e ganho de peso diário. Os resultados de biomassa final do atual experimento aumentaram conforme o acréscimo de indivíduos estocados por unidade de área. Os maiores valores encontrados foram àqueles relacionados ao tratamento com maior densidade de estocagem.

Piaia \& Baldisseroto (2000) obtiveram resultado semelhante em pesquisa com três densidades de estocagem $\left(114,227\right.$ e 454 alevinos. $\left.\mathrm{m}^{-3}\right)$ e crescimento de alevinos de jundiá (Rhamdia quelen). Esses autores observaram que a maior densidade proporcionou a maior biomassa final por volume e sugeriram que, na fase inicial de criação, altas densidades de criação não comprometem o desenvolvimento dessa espécie. Rowland et al. (2005), trabalhando com o efeito da densidade de estocagem $(12,25,50$, 100 e 200 peixes. $\mathrm{m}^{-3}$ ) no desempenho de "silver perch" (Bidyanus bidyanus), não encontraram diferenças significativa no peso final entre as densidades, porém, para biomassa final, os maiores resultados foram obtidos nos tanques-rede que continham maior número de peixes.

A taxa de sobrevivência de pacus (P. mesopotamicus) cultivados em tanques-rede em diferentes densidades de estocagem não foi afetada durante a fase de crescimento e terminação.

Os valores de conversão alimentar dos peixes criados em tanques-rede não diferiram significativamente entre a densidades de estocagem. Gomes et al. (2000) também não 
observaram diferenças na conversão alimentar na criação de larvas de matrinxã (Brycon cephalus) criados em diferentes densidades de estocagem. Em contrapartida, Rowland et al. (2005) verificaram que a conversão alimentar foi influenciada pela taxa de estocagem na criação de Bidyanus bidyaus e encontraram os piores resultados nos tanques-rede contendo 25 e 50 peixes. $\mathrm{m}^{-3}$.

Entre os parâmetros de rendimento de carcaça (Tabela 2), o único que apresentou diferença significativa $(\mathrm{P}<0,05)$ foi o comprimento total, que foi maior nos peixes estocados em menor densidade e não diferiu daqueles cultivados em densidade intermediária.

Respostas semelhantes foram observadas por Faria et al. (2003), que estudaram o rendimento do processamento de pacus ( $P$. mesopotamicus) e encontraram a seguinte composição: $46,73 \%$ de filé sem pele; $16,57 \%$ de cabeça; e $88,98 \%$ de rendimento de carcaça. Esses pesquisadores afirmaram que o alto rendimento de filé alcançado para essa espécie tem correlação inversa com o tamanho da cabeça dos animais.

Benke et al. (2005) também registraram valores semelhantes de rendimento de carcaça $(81,30$ a $84,40 \%)$ quando avaliaram os cortes da carne de pacus (P. mesopotamicus) cultivados em tanque-rede no reservatório de Itaipu, porém observaram que os cortes influenciaram no aproveitamento do filé (22,41 a 24,09\%), pois contemplavam somente a musculatura existente sobre as costelas, ou seja, não incluíam a porção dorsal.

Neste trabalho foram observadas semelhanças de rendimento de filé e superioridade no aporte da carcaça aos resultados obtidos por Burkert et al. (2008), em estudo do processamento de surubins (Pseudoplatystoma sp.) cultivados em tanques-rede. Esses autores salientaram ainda que o rendimento de filé pode ter sido prejudicado pela

Tabela 2 - Rendimento de carcaça de pacus em tanque-rede criados em três densidades de estocagem

\begin{tabular}{|c|c|c|c|c|}
\hline \multirow[t]{2}{*}{ Parâmetro ${ }^{1}$} & \multicolumn{3}{|c|}{ Densidade } & \multirow{2}{*}{$\begin{array}{l}\text { CV } \\
(\%)\end{array}$} \\
\hline & 200 & 300 & 400 & \\
\hline Comprimento total $(\mathrm{cm})$ & $36,95 \mathrm{a}$ & $35,07 \mathrm{ab}$ & $32,57 \mathrm{~b}$ & 2,88 \\
\hline Comprimento padrão (cm) & 32,60 & 30,89 & 29,13 & 3,35 \\
\hline Peso total $(\mathrm{g})$ & 1279,00 & 1131,00 & 911,00 & 12,08 \\
\hline Rendimento de carcaça (\%) & 88,02 & 87,39 & 86,45 & 2,54 \\
\hline Tronco limpo (\%) & 60,92 & 59,92 & 59,31 & 1,60 \\
\hline Filé (\%) & 46,86 & 47,77 & 45,48 & 1,64 \\
\hline Cabeça (\%) & 13,08 & 14,00 & 14,52 & 7,96 \\
\hline Gordura visceral (\%) & 7,02 & 6,84 & 6,45 & 8,75 \\
\hline Índice hepatossomático & 0,96 & 0,99 & 0,92 & 8,89 \\
\hline
\end{tabular}

deposição de gordura, tanto na região celomática quanto na musculatura, pois os exemplares apresentaram pesos superiores a $5 \mathrm{~kg}$, o que colaboraria para o maior acúmulo de gordura. Em contrapartida, o pacu criado em tanque-rede sob diferentes densidades apresentou valores superiores para rendimento de filé e gordura visceral de matrinxã (Brycon cephalus) processada em diferentes classes de peso (Macedo-Viegas et al., 2000).

Essas discrepâncias nos valores de rendimento de carcaça podem ser explicadas pela influência de fatores como espécie, formato anatômico do peixe, peso corporal, tamanho da cabeça, porcentagem de resíduos, eficiência das máquinas filetadoras e/ou destreza do filetador, entre outros (Faria et al., 2003).

Os valores de matéria seca, proteína bruta e matéria mineral dos peixes nesta pesquisa não apresentaram diferença estatística $(\mathrm{P}>0,05)$ entre as densidades de criação (Tabela 3 ). Esse resultado corrobora aquele encontrado por Toko et al. (2007) na criação de Clarias gariepinus e Heterobranchus longifilis em tanques convencionais com diferentes densidades de estocagem. Esses autores não notaram influência do número de indivíduos por unidade de área nos valores de matéria seca, proteína bruta e matéria mineral da carcaça dos animais das duas espécies. El-Sayed (2002), avaliando a densidade de estocagem $(3,5,10,15$ e 20 alevinos. $L^{-1}$ ) na criação de alevinos de tilápia-do-nilo (Oreochromis niloticus), não notou diferença nos teores de proteína bruta e matéria mineral entre as densidades de criação.

Valores equivalentes aos obtidos neste estudo foram relatados por Arbeláez-Rojas et al. (2002) em trabalho no qual avaliaram a composição corporal de tambaquis (Colossoma macropomum) em sistemas de produção intensivo e semi-intensivo e não encontraram diferenças significativas $(\mathrm{P}>0,05)$ nos valores de umidade, proteína bruta e matéria mineral dos peixes.

Tabela 3 - Composição química da carcaça, na matéria natural, de pacus em tanque-rede criados em três densidades de estocagem

\begin{tabular}{lcccc}
\hline Parâmetro $^{1}$ & \multicolumn{3}{c}{ Densidade } & CV \\
\cline { 2 - 4 } & 200 & 300 & 400 & \\
\hline Matéria seca (\%) & 41,28 & 40,88 & 37,30 & 5,80 \\
Proteína bruta (\%) & 19,95 & 19,00 & 18,19 & 4,60 \\
Matéria mineral (\%) & 3,75 & 3,01 & 2,98 & 16,88 \\
Extrato etéreo (\%) & $21,82 \mathrm{a}$ & $19,51 \mathrm{ab}$ & $15,41 \mathrm{~b}$ & 9,97 \\
\hline
\end{tabular}

${ }^{1}$ Médias na mesma linha seguidas de letras distintas diferem entre si pelo teste de Tukey a $5 \%$.

$\mathrm{CV}=$ coeficiente de variação. 
Na composição química dos peixes, o teor de extrato etéreo foi a única variável que sofreu influência significativa $(\mathrm{P}<0,05)$ da densidade de estocagem. Os menores valores para esse parâmetro foram encontrados nos animais estocados em maior número, que diferiram daqueles cultivados em menor densidade de estocagem e foram equivalentes à densidade intermediária. Em contrapartida, Arbeláez-Rojas et al. (2002) observaram menor deposição de lipídios em tambaquis (C. macropomum) produzidos em sistemas de produção intensivo $(1,4 \%)$ e semi-intensivo $(2,41 \%)$. Esses resultados comprovam a influência do sistema de criação na composição bromatológica dos animais.

Os lipídios são essenciais para o metabolismo nos peixes (Fujimoto et al., 2007b) e possuem funções energéticas, estruturais, hormonais, entre outras (Haliloglu et al., 2003). Entretanto, em peixes de tamanho comercial, a deposição de lipídios é indesejável e deve ser mantida em nível que não afete as características organolépticas da carne (Meurer et al., 2003b).

Os animais cultivados em tanque-rede em diferentes densidades de estocagem não apresentaram diferenças estatísticas nos valores de glicose, hemoglobina, hematócrito, eritrócito, volume corpuscular médio, concentração de hemoglobina corpuscular média e hemoglobina corpuscular média (Tabela 4).

Embora a diferença não tenha sido significativa, os valores glicêmicos foram superiores àqueles encontrados por Brandão et al. (2004), 51-65 mg.dL ${ }^{-1}$, em estudo com juvenis de tambaqui (Colossoma macropomum) durante a recria em tanques-rede com três densidades de estocagem $\left(200,300,400\right.$ e 500 peixes. $\left.\mathrm{m}^{-3}\right)$. É possível que essa hiperglicemia esteja relacionada ao manejo de captura dos animais e ao transporte até o local foram realizadas as amostragens sanguíneas.

Segundo Urbinati \& Carneiro (2004), para atender às demandas energéticas dos organismos em situações

Tabela 4 - Parâmetros hematológicos de pacus em tanque-rede criados em três densidades de estocagem

\begin{tabular}{lcccc}
\hline Parâmetro $^{1}$ & \multicolumn{3}{c}{ Densidade } & CV \\
\cline { 2 - 4 } & 200 & 300 & 400 & \\
\hline Glicose $\left(\mathrm{mg}^{\prime} \mathrm{dL}^{-1}\right)$ & 106,55 & 99,95 & 105,28 & 25,22 \\
Hemoglobina $\left(\mathrm{g} . \mathrm{dL}^{-1}\right)$ & 9,98 & 10,09 & 9,42 & 12,00 \\
Hematócrito $(\%)$ & 42,93 & 41,00 & 40,13 & 12,48 \\
Eritrócito $\left(10^{6} \mu \mathrm{L}\right)$ & 1,88 & 1,85 & 1,85 & 15,05 \\
$\begin{array}{l}\text { Volume corpuscular médio }\left(\mu^{3}\right) \\
\text { Concentração de hemoglobina }\end{array}$ & 231,81 & 224,21 & 219,22 & 16,17 \\
$\begin{array}{l}\text { corpuscular média (\%) } \\
\text { Hemoglobina corpuscular }\end{array}$ & 51,14 & 52,27 & 22,64 & 15,69 \\
média (pg) & & & & \\
\hline 1(P>0,05); CV = coeficiente de variação. & & & \\
\hline
\end{tabular}

adversas, as catecolaminas atuam diretamente no fígado, estimulando a glicogenólise e transformando o glicogênio em glicose. Como os pacus estavam em jejum por um período de 24 horas no momento das coletas, as reservas hepáticas foram as prováveis fontes de glicose para sanar as demandas energéticas momentâneas.

O número total de eritrócitos não variou significativamente entre as densidades de criação e apresentou valores muito coesos. Essa proximidade nos resultados pode ser comprovada pelas médias de hemoglobina e hematócrito, que também não apresentaram diferenças estatísticas e foram semelhantes entre as densidades de estocagem utilizadas. Os padrões de eritrócitos para o P. mesopotamicus em cativeiro, segundo Tavares-Dias \& Moraes (2004), são em torno de 1,63 a $3,13 \times 10^{6} \mu \mathrm{L}$, como observado neste estudo.

Montero et al. (1999) estudaram o efeito da densidade de estocagem (30, 90 e 120 peixes/tanque de $250 \mathrm{~L}$ ) nos parâmetros bioquímicos e fisiológicos de "gilthead seabream" (Sparus aurata) e verificaram diferenças significativas nos valores de eritrócitos, hemoglobina e hematócrito nos animais estocados em maior número. Os autores relataram que esse acréscimo eritrocitário refere-se ao aumento da capacidade sanguínea em transportar o oxigênio durante períodos de demanda energética elevados. Em contraponto, os pacus não apresentaram alterações em seus parâmetros eritrocitários, o que permite afirmar que estavam em estado de higidez.

A concentração de hemoglobina e a porcentagem de hematócrito não diferiram estatisticamente e permaneceram dentro dos padrões adequados para a espécie (TavaresDias \& Moraes, 2004). O hematócrito é expresso como um volume percentual das células empilhadas no sangue total após a centrifugação. Neste experimento, os valores permaneceram entre 40 e $43 \%$, mas a maioria das espécies de animais domésticos tem hematócrito variando entre 38 e $45 \%$, com média de 40\% (Swenson, 1996).

Fujimoto et al. (2007a), avaliando os parâmetros sanguíneos de pacus (P. mesopotamicus) alimentados com dietas suplementadas com cromo trivalente em duas densidades de estocagem (4 e $20 \mathrm{~kg}$ de peixe. $\mathrm{m}^{-3}$ ), encontraram diferenças estatísticas no eritrócito dos animais entre as densidades de criação, porém as médias de hematócrito foram semelhantes entre si.

Os índices eritrocitários volume corpuscular médio, hemoglobina corpuscular média e concentração de hemoglobina corpuscular média não apresentaram diferenças significativas entre as densidades de criação e permaneceram próximos dos valores encontrados por 
Ranzani-Paiva et al. (1999). Resultados similares aos deste trabalho foram reportados por Montero et al. (1999), que, em trabalho com juvenis de Sparus aurata, não encontraram influência da densidade de estocagem nesses parâmetros.

\section{Conclusões}

A maior densidade de estocagem resulta em menor peso final dos pacus (P. mesopotamicus) sem interferir no rendimento de carcaça, porém apresenta maior biomassa final com menor deposição de lipídeos. As médias eritrocitárias permanecem dentro dos valores recomendados para a espécie e não se alteram com variações na densidade de estocagem. Os níveis sanguíneos de glicose não são influenciados pela densidade de criação na unidade produtiva.

\section{Agradecimentos}

À Itaipu Binacional e à UNIOESTE/Campus de Toledo, que, por intermédio do Convênio de Cooperação TénicoFinanceiro sob $n^{\circ} 4500003031$ firmado entre as partes, possibilitaram a realização deste trabalho.

\section{Referências}

ARBELÁEZ-ROJAS, G.A.; FRACALOSSI, D.M.; FIM, J.D.I. Composição corporal de tambaqui, Colossoma macropomum, e matrinxã, Brycon cephalus, em sistemas de cultivo intensivo, em igarapé, e semi-intensivo, em viveiros. Revista Brasileira de Zootecnia, v.31, n.3, p.1059-1069, 2002. ASSOCIATION OF OFFICIAL ANALYTICAL CHEMISTRY AOAC. Official methods of analysis of the AOAC. 18.ed. Gaithersburg: 2005.

BENKE, B.; BOMBARDELLI, R.A.; SANCHES, E.A. et al. Características morfométricas e rendimento de cortes da carne do pacu (Piaractus mesopotamicus) cultivado em tanques-rede no reservatório de Itaipu. In: CONGRESSO BRASILEIRO DE ENGENHARIA DE PESCA - CONBEP, 14., 2005, Fortaleza. Anais... Fortaleza: 2005. (CD-ROM).

$\mathrm{BOYD}, \mathrm{C}$. Water quality in ponds for aquaculture. Alabama: Birmingham Publishing, 1990. 482p.

BRANDÃO, F.R.; GOMES, L.C.; CHAGAS, E.D. et al. Densidade de estocagem de juvenis de tambaqui durante a recria em tanquesrede. Pesquisa Agropecuária Brasileira, v.39, n.4, p.357-362, 2004.

BUENO, G.W.; MARENGONI, N.G.; GONÇALVES JR., A.C. et al. Estado trófico e bioacumulação do fósforo total no cultivo de peixes em tanques-rede na área aquícola do reservatório de Itaipu. Acta Scietiarum.Biological Sciences, v.30, n.3, p.237-243, 2008.

BURKERT, D.; ANDRADE, D.R.; SIROL, R.N. et al. Rendimento do processamento e composição química de filés de surubim cultivado em tanques-rede. Revista Brasileira de Zootecnia, v.37, n.7, p.1137-1143, 2008.

CAVERO, B.A.S.; PEREIRA-FILHO, M.; ROUBACH, R. et al. Biomassa sustentável de juvenis de pirarucu em tanques-rede de pequeno volume. Pesquisa Agropecuária Brasileira, v.38, n.6, p.723-728, 2003.

COLLIER, H.B. The standardization of blood haemoglobin determinations. Canadian Mediacal Association Journal, Vancouver, v.50, p.550-552, 1944

COULIBALY, A.; OUATTARA, I.N.; KONÉ, T. et al. First results of floating cage culture of the African catfish Heterobranchus longifilis Valenciennes, 1840: Effect of stocking density on survival and growth rates. Aquaculture, v.263, p.61-67, 2006.

EL-SAYED, A.F.M. Effects of stocking density and feeding levels on growth and feed efficiency of nile tilapia (Oreochromis niloticus L.) fry. Aquaculture Research, v.33, p.621-626, 2002.

FARIA, R.H.S.; SOUZA, M.L.R.; WAGNER, P.M. et al. Rendimento do processamento da tilapia do Nilo (Oreochroms niloticus Linnaeus, 1757) e do pacu (Piaractus mesopotamicus Holmberg, 1887). Acta Scientiarum. Animal Sciences, v.25, n.1, p.21-24, 2003.

FUJIMOTO, R.Y.; CASTRO, M.P.; MORAES, F.R. et al. Parâmetros sanguíneos de pacu Piaractus mesopotamicus (Holmberg, 1887) alimentados com dietas suplementadas com cromo trivalente em duas densidades de estocagem. Acta scientiarum. Animal sciences, v.29, n.4, p.465-471, 2007a.

FUJIMOTO, R.Y.; CASTRO, M.P.; HONORATO, C.A. et al. Composição corporal e eficiência de utilização de nutrientes por pacus alimentados com ração suplementada com cromo trivalente. Pesquisa Agropecuária Brasileira, v.42, n.12, p.1763-1768, 2007b.

GOMES, L.C.; BALDISSEROTTO, B.; SENHORINI, J.A. et al Effect of stocking density on water quality, survival, and growth of larvae of matrinxã, Brycon cephalus (Characidae), in ponds. Aquaculture, v.183, p.73-81, 2000.

GOLDENFARB, P.B.; BAYIR, A.; NECDET SIRKECIOGLU, A. et al. Reproducibility in the hematology laboratory: the microhematocrit determination. American Journal of Clinical Pathology, v.56, p.35-39, 1971.

HALILOGLU, H.I. et al. Comparisons of fatty acid composition in some tissues of rainbow (Oncorhynchus mykiss) living in seawater and freshwater. Food Chemistry, v.86, p.55-59, 2003.

IGUCHI, K.; OGAWA, K.; NAGAE, M. et al. The influence of rearing density on stress response and disease susceptibility of ayu (Plecoglossus altivelis). Aquaculture, v.202, p.515-523, 2003.

MACEDO-VIEGAS, E.M.; SCORVO, C.M.D.F.; VIDOTTI, R.M. et al. Efeito das classes de peso sobre a composição corporal e o rendimento de processamento de matrinxã (Brycon cephalus). Acta Scientiarum, v.22, n.3, p.725-728, 2000.

MARENGONI, N.G. Produção de tilápia do Nilo Oreochromis niloticus (linhagem chitralada), cultivada em tanques rede, sob diferentes densidades de estocagem. Archivos de Zootecnia, v. 55, p.127-138, 2006

MEURER, F.; HAYASHI, C.; BOSCOLO, W.R. et al. Digestibilidade aparente de alguns alimentos protéicos pela tilápia do Nilo (Oreochromis niloticus). Revista Brasileira de Zootecnia, v.32, n.6, p.1801-1809, 2003b.

MONTERO, D.; IZQUIERDO, M.S.; TORT, L. et al. High stocking density produces crowding stress altering some physiological and biochemical parameters in gilthead seabream, Sparus aurata, juveniles. Fish Physiology and Biochemistry, v.20, p.53-60, 1999

ONO, E.A.; KUBITZA, F. Cultivo de peixes em tanques-rede. 2.ed. Jundiaí: F. Kubitza, 1999. 68p.

PIAIA, R.; BALDISSEROTO, B. Densidade de estocagem e crescimento de alevinos de jundiá Rhamdia quelen (Quoy \& Gaimard, 1824). Ciência Rural, v.30, p.509-513, 2000.

RANZANI-PAIVA, M.J.T.; SALLES, F.; EIRAS, J. et al. Análises hematológicas de curimbatá (Prochilodus scrofa), pacu (Piaractus mesopotamicus) e tambaqui (Colossoma macropomum) das estações de piscicultura do instituto de pesca, estado de São Paulo. Boletim do Instituto de Pesca, v.25, p.77-83, 1999. 
ROWLAND, S.J.; MIFSUD, C.; NIXON, M. et al. Effects of stocking density on the performance os the Australian freshwater silver perch (Bidyanus bidyanus) in cages. Aquaculture, v.253, p.301-308, 2005.

SCHALCH, S.H.C.; BELLO, M.A.A.; SOARES, V.E. et al. Eficácia do diflubenzuron no controle de Dolops carvalhoi (Crustácea: Branchiura) em jovens pacus Piaractus mesopotamicus (Osteichthyes: Characidae) naturalmente infectados. Acta Scietiarum. Animal Science, v.27, n.2, p.297-302, 2005 .

SIPAÚBA-TAVARES, L.H.S. Limnologia aplicada a aqüicultura. Jaboticabal: Funep, 1995. 72p.

SILVA, P.C.; KRONKA, S.N.; SIPAÚBA-TAVARES, L.H. et al. Desempenho produtivo de tilápia do Nilo (Oreochromis niloticus L.) em diferentes densidades e trocas de água em "raceway". Acta Scientiarum, v.24, n.4, p.935-941, 2002.

SWENSON, M.J. Propriedades fisiológicas e constituinte químicos e celulares do sangue. In: SWENSON, M.J.; REECE, W.O.
(Eds.) Dukes - Fisiologia dos animais domésticos. Rio de Janeiro: Ganabara Koogan, 1996. 856p.

TAVARES-DIAS, M.; MORAES, F.R. Hematologia de peixes teleósteos. Ribeirão Preto: Villimpress Complexo Gráfico, 2004. $144 \mathrm{p}$.

TOKO, I.; FIOGBE, E.D.; KOUKPODE, B. et al. Rearing of African catfish (Clarias gariepinus) and vudu catfish (Heterobranchus longifilis) in traditional fish ponds (whedos): Effect of stocking density on growth, production and body composition. Aquaculture, v.262, p.65-72, 2007.

URBINATI, E.C.; GONÇALVES, F.D. Pacu (Piaractus mesopotamicus). In: BALDISSEROTO, B.; GOMES, L.C. Espécies nativas para piscicultura no Brasil. Santa Maria: Editora UFSM, 2005. 470p.

URBINATI, E.C.; CARNEIRO, P.C.F. Práticas de manejo e estresse dos peixes em piscicultura. In: CYRINO, J.E.P.; URBINATI, E.C.; FRACALOSSI, D.M. et al. (Eds.) Tópicos especiais em piscicultura de água doce tropical intensiva. São Paulo: TecArt, 2004. p.533. 